\section{UAV Workflow Optimization for the Acquisition of High-Quality Photogrammetric Point Clouds in Forestry}

Gl_Forum 2016, Vol.1

Page: 72-84

Full Paper

Corresponding Author:

stuart.krause@hnee.de

DOI: 101553/giscience2016_01_s72

\author{
Stuart Krause, Fabian Hartmann and Jan-Peter Mund \\ Eberswalde University of Sustainable Development, Germany
}

\begin{abstract}
Three-dimensional modelling using photogrammetric point clouds derived from UAV-based aerial imagery is currently a popular topic in the scientific community. In particular, the use of image-based point clouds to enhance and update LiDAR DSMs is of growing interest in forest environments, i.e. as a future forest inventory method. Thanks to very high resolution imagery acquired via low-altitude UAV optical sensor payloads, very dense and accurate photogrammetric point clouds can be reconstructed through a triangulation process by means of photogrammetry software. In order to validate the use of image-based point clouds for their potential use in operational forestry, further comparison studies with LiDAR DSMs are being carried out by various research institutions. The acquisition of UAV-based aerial imagery, with the aim of producing accurate photogrammetric point clouds, though cost-effective, is not without its challenges. Due to constraints regarding power capacity and fair weather windows, we came to develop an effective image acquisition workflow with an emphasis on precision flight planning. The aim of this paper is to explore the process of UAV-based aerial imagery acquisition for the purpose of producing photogrammetric point clouds, as well as to give an overview of the initial stages of our research. With the aid of an image acquisition workflow that is adaptable to various field conditions, technical failures and precision flight planning, we estimate that the acquisition of aerial imagery for point cloud production will become more efficient as well as more precise, and in turn influence the accuracy of the 3D-modelling of forested areas.
\end{abstract}

\title{
Keywords:
}

UAV, point clouds, photogrammetry, forestry, flight planning

\section{Introduction}

The utilization of Unmanned Aerial Vehicles (UAVs) to acquire very high resolution $(<0.1 \mathrm{~m}$ GSD) aerial imagery is becoming more and more relevant in various sectors, including forestry. Today, high density point clouds originating from UAV-based aerial imagery can be acquired cost-effectively thanks to relatively low operational costs (Hollaus, 2014). When combined with LiDAR datasets, such point clouds improve the potential for more precise modelling and interpretation (Basgall, 2013) of particular forest parameters and Digital Surface Models (DSMs) of forest environments. Due to the similarity 
of photogrammetric point clouds (PPCs) originating from aerial imagery to point clouds derived from LiDAR, digital aerial imagery is of increasing interest for forest managers to estimate tree canopy heights (White et al., 2013).

The acquisition of UAV-based aerial imagery, with the aim of creating PPCs, though costeffective in comparison to Airborne Laser Scanning (manned aircraft), is not without its challenges. The aim of this paper is to explore the process of UAV-based aerial imagery acquisition for the purpose of producing PPCs, as well as to give an overview of the initial stages of our research. With the aid of an image acquisition workflow that is adaptable to various field conditions, technical failures, and precision flight planning, we estimate that the acquisition of aerial imagery for point cloud production will become more efficient as well as more precise, and in turn influence the accuracy of the 3D-modelling of forested areas.

\section{Photogrammetric Point Clouds}

Traditional photogrammetry has been implementing stereoscopy for decades and is an operational method to simultaneously observe images taken from two different camera angles (Campbell \& Wynne, 2011). Viewing objects stereoscopically enables a threedimensional stereo model, which increases the accuracy of depth perception (Wolf et al., 2014), allowing the user to measure the heights of, for example, buildings or trees. During the rise of digital camera technology, developments in stereo matching allowed for digital 3D reconstruction by triangulation using intrinsic and extrinsic orientation of cameras to find corresponding pixels in image pairs (Hirschmüller, 2011). The reconstruction of image pairs through triangulation leads to the production of a point cloud dataset which is composed of points possessing XYZ coordinates. Derived from high-resolution imagery, PPCs can give a detailed and accurate 3D representation of surfaces. Objects enhanced with radiometric pixel values can even produce photorealistic point clouds (Colditz et al., 2005). Today, the SemiGlobal Matching (SGM) algorithm is implemented by various photogrammetry software packages and offers an effective balance between processing-time and accuracy (Hirschmüller, 2011).

PPCs are similar to LiDAR in the sense that vertical information can be represented in a dataset (White et al., 2013), but with an added radiometric aspect (e.g. RGB) derived from high- to very-high-resolution imagery. Combining PPCs with LiDAR datasets is a promising method for forest height measurement (Lisein et al., 2013) and can also be implemented to improve species classification (St-onge et al., 2015).

\section{LiDAR vs Photogrammetric Point Clouds}

LiDAR is capable of creating high-quality DSMs with minimal occlusion issues. LiDAR pulses can reach through to the canopy floor (Lisein et al., 2013; White et al., 2013), accessing height information, even of a forest stand's understorey. Although, due to occlusion (Miri et al., 2013), PPCs lack understorey information, it is possible, for example by using oblique camera angles, to reach sections below the forest canopy (see Figure 2). In a study conducted by St-onge et al. (2015), it was shown that the 3D-reconstruction of PPCs (unlike LiDAR images) was influenced by varying forest structures such as dense and open 
canopies, as well as species composition. In the same study, St-onge et al. further state that the image-matching process produced a point density three to five times higher than the first-return density obtained using LiDAR.

The fact that point clouds derived from aerial or UAV-based imagery can achieve such a high density does not mean that PPCs will replace those derived from LiDAR in the near future. PPCs, when combined with LiDAR datasets (Basgall, 2013), can increase point cloud pixel density, creating a promising tool for forest research and management. Due to the high costs of acquiring LiDAR datasets, LiDAR manned flights are not flown at regular intervals. PPCs, on the other hand, can potentially have a high temporal resolution, although they have a lower spatial survey capacity. Due to these limiting factors, PPCs can be utilized to update particular regions of LiDAR datasets on a regular basis until the next manned LiDAR mission is flown. More comparative studies between LiDAR and PPC estimates of forest inventory attributes are required for varying forest structure types in order to assess the potential and limitations (White et al., 2013) of this technology. It is our assumption that the success of PPC quality is reliant on the image acquisition process, in particular the implementation of precision flight planning.

\section{$3 \quad$ Flight planning}

In order to achieve sufficient frontal (e.g. 85\%) and side (e.g. 65\%) overlap of imagery, acquisition is most effectively implemented using a predetermined automatized flight grid. With the ability to fly autonomously, a UAV can acquire images at precise geographical locations (GPS/GLONASS), thus ensuring proper area coverage. UAV-based aerial imagery acquired through manual flight, however, is very challenging and is not recommended for forest inventory purposes, as inadequate coverage can result in low-quality image mosaics and PPCs derived from such datasets.

Due to potential technical and meteorological obstacles, the process of collecting accurate image datasets with adequate image overlap must be organized in such a way that flight plans can be adjusted spontaneously without losing calculation and image mosaicking integrity. We gained experience through various flight campaigns using a professionally-manufactured Octocopter (Octagon - Soleon), implementing various image-sensor payloads; we are also utilizing a consumer-grade Quadrocopter (Phantom 3 Professional-DJI). Through an iterative process, we developed a comprehensive workflow procedure with the aim of facilitating more standardized empirical research in the near future.

We are currently developing an automated method to improve the accuracy of the flight-plan parameter calculations required for creating the flight path grid. A prominent feature of this process is to predict image footprints on the ground from nadir and oblique imagery. We found that such an automated process is critical, typically when a flight plan requires alterations in the field or even during flight. The inability to deal with unexpected factors such as high winds or a sudden decrease in solar illumination could influence image quality, which can in turn affect the accuracy and density of the PPCs (Rosnell et al., 2012). During our initial trials, low-quality imagery and insufficient overlap resulted in faulty image calibration, and decreased point cloud densities. In addition to providing sufficient overlap 
and high-resolution imagery, a successful flight plan also preserves the UAV's power capacity and saves the amount of time spent in the field during data-collection campaigns.

Various open source and proprietary software packages are available that can automatically calculate flight planning grids, depending on typical aerial photography parameters. These parameters include for example, camera focal length, sensor size, forward and side image overlap, and flying altitude (Wolf et al., 2014). Flight planning software can not only automatically generate a flight grid, but also function as a ground control station for the UAV, where real-time flight paths can be monitored on a laptop, tablet or even smartphone. Unfortunately, typical automatically-generated flight plans are often limited to the requirements of UAV hobbyists, where it is adequate to implement a Google Earth base map in order to confirm geographical locations, and the production of generic flight plan grids using flight planning software. For forestry applications, however, it is useful to be able to pinpoint precise, differential GNSS-derived 3D-geolocations in order to plan proper image coverage of the area of interest as well as account for low oblique imagery ground coverage. We have found that flight-planning software that allows for the importation of flight planning grids, along with other flight parameters in the form of a comma separated value (csv) or similar editable text file, can increase the efficiency and accuracy of a flight plan (see Table 1). A flight plan grid can be manually calculated with a GIS in order to ensure the accuracy of flight paths, and can be adapted to flight-planning software through scripting. Using this method, precise geographical coordinates (e.g. WGS 1984, decimal degrees) can be allocated to each camera trigger position, and image overlap and projection can be confirmed.

Because the aerial imagery acquisition process has already been in operation for decades, we recommend a comprehensive study of traditional flight-planning techniques, as opposed to relying exclusively on the UAV-based "Black Box" flight plan generation applications which are incorporated into currently available UAV flight-planning software. The initial manual calculation of flight plan parameters is especially useful when planning for the acquisition of oblique imagery, as most software we have encountered does not account for the projections of oblique camera angles during flight-plan grid generation. Through empirical tests, we discovered that in tree stands with relatively open canopies, it was possible to gather data from tree stems as well as understorey vegetation by using oblique imagery (see Figure 2). In the near future, specially adapted flight plan grids modified for oblique imagery acquisition as well as varying terrain will be empirically tested on controlled forest stands.

Table 1: Example of terrain-adapted flight plan parameters in csv file format.

\begin{tabular}{|c|l|l|l|l|l|l|l|c|c|}
\hline WP & Latitude & Longitude & Radius & Alt. & $\begin{array}{l}\text { Climb Rate } \\
{[\mathbf{m} / \mathbf{s} \mathbf{x} \text { 10] }}\end{array}$ & $\begin{array}{l}\text { WP } \\
\text { Event }\end{array}$ & $\begin{array}{l}\text { Headi } \\
\text { ng }\end{array}$ & $\begin{array}{l}\text { Speed } \\
\text { [m/s x } \\
\text { 10] }\end{array}$ & ${\begin{array}{l}\text { Gim } \\
\text { bal }^{\circ}\end{array}}$ \\
\hline 1 & 52.8193384 & 13.7920515 & 10 & 79 & 30 & 100 & 360 & 25 & 90 \\
\hline 2 & 52.8195093 & 13.7920416 & 10 & 79 & 30 & 100 & 360 & 25 & 90 \\
\hline 3 & 52.8196802 & 13.7920318 & 10 & 81 & 30 & 100 & 360 & 25 & 90 \\
\hline 4 & 52.8198511 & 13.792022 & 10 & 78 & 30 & 100 & 360 & 25 & 90 \\
\hline 5 & 52.8193384 & 13.7920515 & 10 & 79 & 30 & 100 & 360 & 25 & 90 \\
\hline
\end{tabular}


We are also experimenting with the use of a time-lapse function, which is possible on many modern consumer-grade cameras. With this automatized acquisition method, the time between images in a flight planning grid is calculated and the camera is set accordingly. The number of waypoints (camera trigger events) required is reduced drastically when a camera's time-lapse function is used, and the amount of data sent to the UAV's flight controller is minimized. The efficiency of the flight campaign is thereby increased when consecutive flight plans are uploaded while the UAV is airborne. Furthermore, the possibility of a failed waypoint event or slow camera trigger is eliminated. This method does, however, create large amounts of unused imagery, which can take up valuable storage space, and require increased data management.

In order to determine the time between images, an "Intervalometer" value can be calculated with regards to the desired flight speed and frontal overlap, as well as the width of the image on the ground (see Formula 1). Figure 1 depicts the relationship between the Intervalometer value and frontal overlap. Frontal image overlap is typically estimated at $>85 \%$ and is essential to ensure adequate pixel-matching during the point cloud production process. Frontal overlap values to the left of the red line represent inadequate image overlap. Values in microseconds may be rounded down; exact values could be achieved depending on a camera's scripting capabilities.

\section{Formula 1:}

$$
I=D_{y} * \frac{\left(\frac{1-O_{f}}{100}\right)}{S}
$$

$\boldsymbol{I}=$ Intervalometer; $\boldsymbol{O}_{\boldsymbol{f}}=$ Frontal Overlap $(\%) ; \boldsymbol{D}_{\boldsymbol{y}}=$ Ground Image Width $(\mathrm{m}) ; \mathbf{S}=$ Flight Speed $(\mathrm{m} / \mathrm{s})$ 


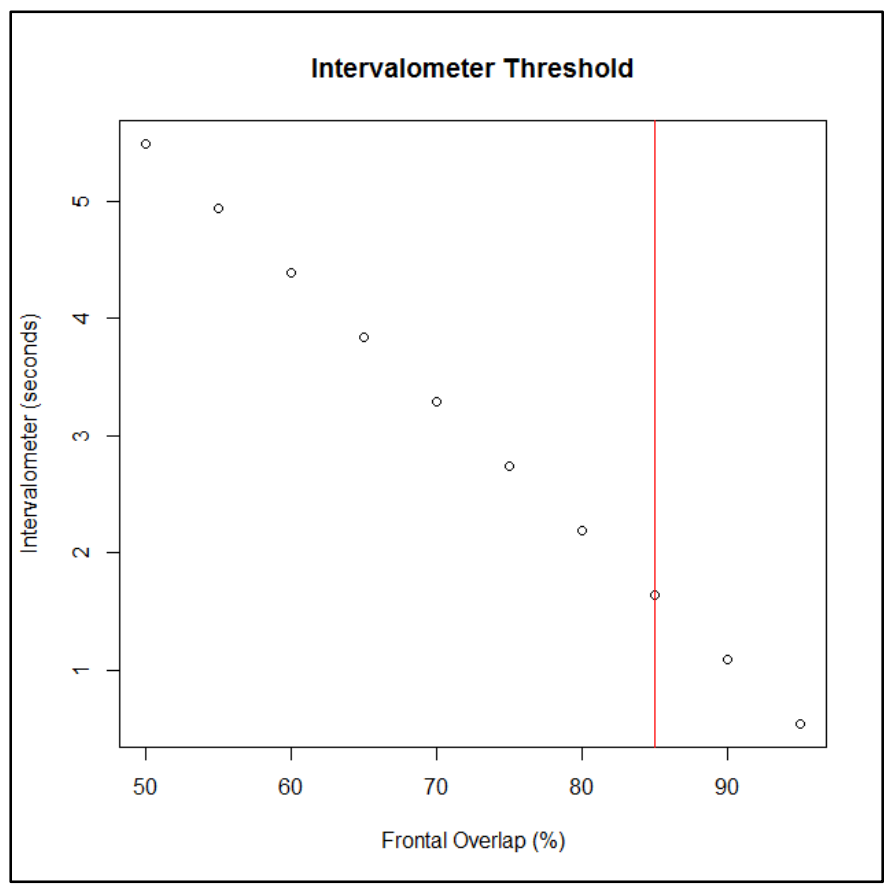

Figure 1: Intervalometer threshold based on a flying altitude of $80 \mathrm{~m}$, flight speed of $5 \mathrm{~m} / \mathrm{s}$, and a Sony A7r camera $(35 \mathrm{~mm})$. Frontal overlap values to the left of the red line represent inadequate image overlap.

\section{Physical and Meteorological Effects on Image Quality and Flight Planning}

Meteorological and physical effects such as clouds, fog, humidity, wind, snow cover and solar elevation have a considerable effect on the UAV and mounted sensors; they can inhibit the stability of a UAV and/or affect sensor payloads and image quality. To deal with such uncontrollable factors that might arise during a mission, the flight plan can be altered, and camera settings can be adapted. However, we did find in relation to the latter that in some cases the pre-processing of images was required.

One of the benefits of UAV image acquisition over that of satellite and manned aircraft is that civilian UAVs usually fly below cloud cover, thus increasing the possibilities of carrying out flight campaigns as well as reducing the extent of image pre-processing, e.g. for atmospheric correction. In some respects, cloud cover can actually improve image quality, due to a more even distribution of diffuse or scattered solar emissions. The sun's rays, particularly in winter (northern hemisphere), can, however, decrease the potential for oblique imagery acquisition, casting long shadows, as well as illuminating the southern side of tree crowns (see Figures 2 and 3). Areas shadowed by trees can also affect image quality and cause height artifacts in the resulting point cloud (Rosnell \& Honkavaara, 2012). Shadowing problems can, however, be overcome to a certain extent through pre-processing (Shahbazi, Sohn, Théau, \& Ménard, 2015). Furthermore, shadow positioning will vary throughout the day, which could prove difficult when combining images from various missions. 


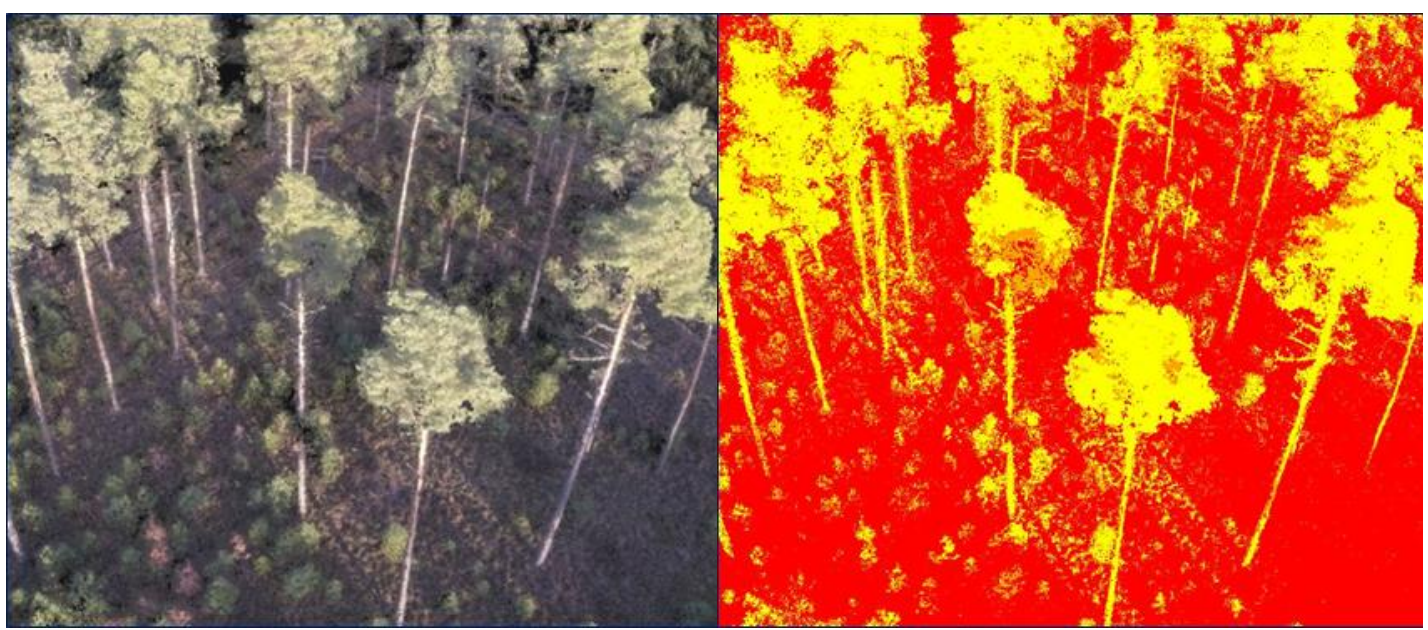

Figure 1: Photorealistic photogrammetric point cloud, with shadow effects of a low altitude winter sun. Realistic RGB colours originate from radiometric pixel values

Figure 2: Classified shadow matrix: red = shadows, orange $=$ semi-shade, yellow $=$ direct sunlight.

In the event of thick cloud cover, fog or humidity, underexposure can pose problems with regard to sensor configuration. Underexposure due to a thick cloud cover can be compensated for with most consumer-grade cameras by using a slower shutter speed $(<1 / 800$ s), yet can result in a reduction in image quality if a higher ISO setting ( $>800$ ISO) is required. A slower shutter speed could also result in image blur when flight speeds greater than $5 \mathrm{~m} / \mathrm{s}$ are to be maintained. We have found that a slower shutter speed is possible when slower flight plan speeds $(<5 \mathrm{~m} / \mathrm{s})$ are implemented, but it can be problematic when mapping larger areas due to power limitations. Ideally, a balance between flight speed, shutter speed, focal length and flying altitude should be attained in order to achieve typical image blur standards $(<0.05 \mathrm{~mm})$.

The blurring of images can be estimated using a formula based on flight speed, shutter speed and scale (see Formula 2). The scale is a function of the camera's focal length and flying altitude. Figure 4 shows the influence of shutter speed on the estimated image blur. Image blur values below the red line $(0.05 \mathrm{~mm})$ are deemed acceptable.

\section{Formula 2:}

$$
B=S * \frac{\left(\frac{1}{t}\right)}{m} * 1000
$$

$\boldsymbol{B}=$ Image Blur; $\boldsymbol{S}=$ Flight Speed $\boldsymbol{t}=$ Shutter Speed $\boldsymbol{m}=$ Scale 


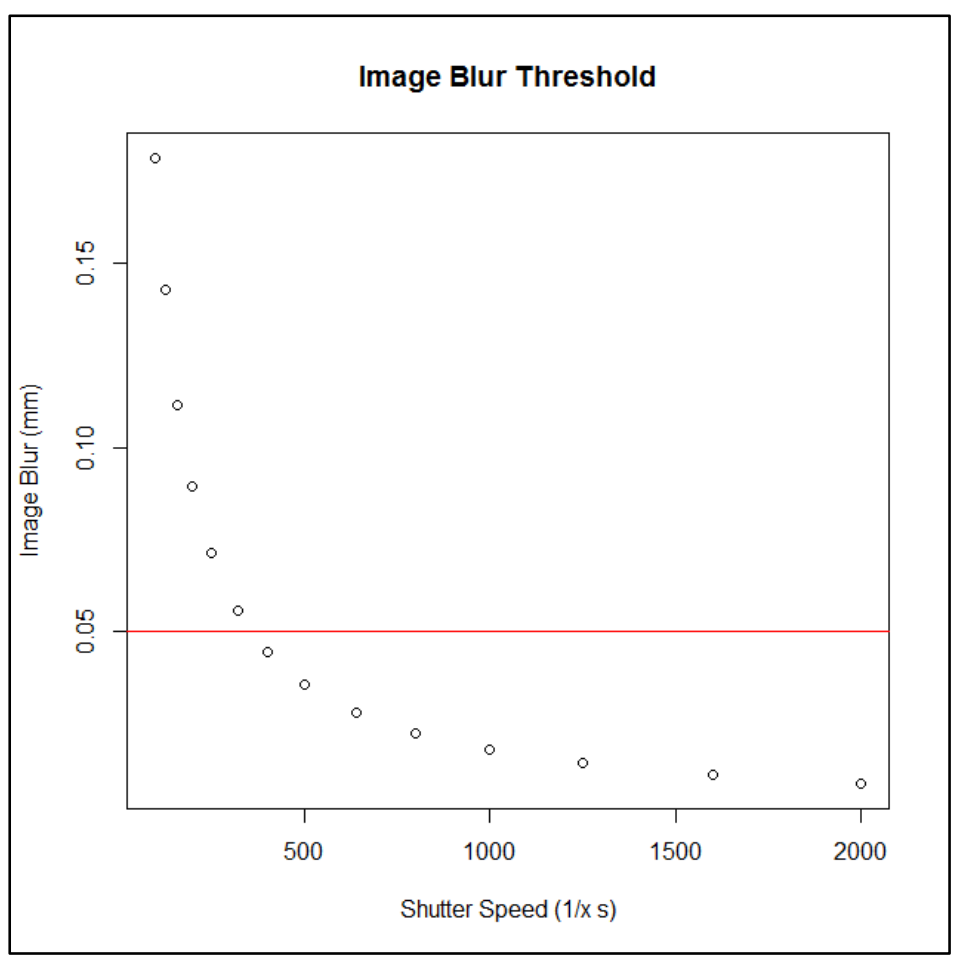

Figure 4: Image Blur threshold based on a flying altitude of $80 \mathrm{~m}$, flight speed of $5 \mathrm{~m} / \mathrm{s}$, using a Sony A7r camera $(35 \mathrm{~mm})$. Image blur values below the red line $(0.05 \mathrm{~mm})$ are deemed acceptable.

Flying in high winds $(>8 \mathrm{~m} / \mathrm{s})$ is not only unsafe, but can also affect flight plan accuracy and hinder the image-matching process due to, for example, moving tree crowns, unstable and tilted UAV positons above the image nadir position, as well as problems with automatic gimbal stabilization. Furthermore, the UAV has to expend more energy in order to remain stable and search for waypoints (see Figure 5). Such factors can affect the geographical positioning and gimbal angle as well as account for uneven overlap. Under more extreme wind conditions, multicopter frontal propellers may appear in the image frame. A reduction of wind influence can be accomplished when flight planning grid lines are programmed in line with the horizontal wind direction, thus reducing the effects of crosswinds. This method, is, however, not foolproof, and in adverse wind conditions the avoidance of flight plan grids is recommended. Compensation for wind can also be accomplished by increasing the precision radius of waypoints (see Table 1), although this will decrease GPS/GLONASS accuracy. 


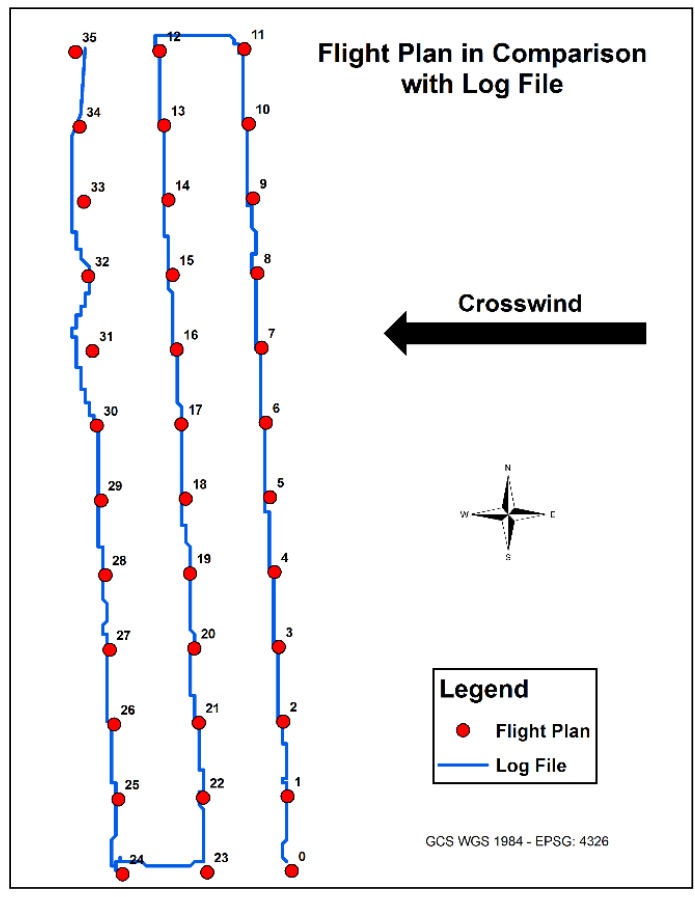

Figure 5: Predetermined flight plan in comparison with the Octocopter log file

Point cloud quality can be poorer in snow-covered areas (Rosnell et al., 2012) as homogeneous surfaces can affect the accuracy of the Automatic Aerial Triangulation (Pix4D Support) process. Snow on tree crowns and stems could also affect the geometrical accuracies of point clouds. Furthermore, based on our experience, snow cover can cause overexposure in images and compensation could decrease the illuminance of trees and vegetation, thus increasing pre-processing workloads.

\section{Workflow Optimization}

Taking into account constraints regarding power capacity, fair weather windows, and even legislation, we developed an effective workflow in order to optimize time spent in the field. The acquisition of UAV-based aerial imagery for the purpose of producing high-density point clouds is essentially an iterative process which begins with, and often returns to, flight planning. In a perfect field campaign where no influencing factors come into play, the flight plan is implemented through various flight missions. After each mission, image quality and coverage are verified, and an image calibration test using photogrammetry software is carried out before leaving the field. A perfect field campaign, however, is a rarity, and the capacity to respond rapidly to unexpected occurrences is paramount. Figure 6 depicts the iterative workflow developed to deal with situations that could arise during a flight campaign. Influences and process directions are shown with black arrows; the red arrows depict a return due to failure. The thick red arrow represents leaving the field. 
Krause et al

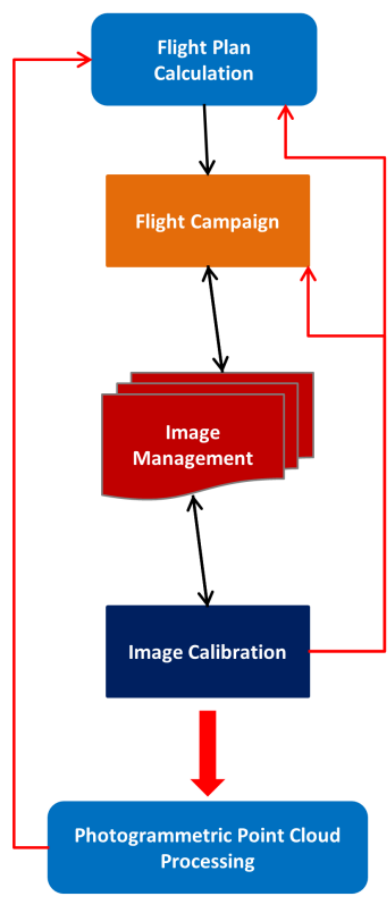

Figure 6: UAV image acquisition iterative workflow

Certain stages, if deemed unsuccessful, are improved by repeating the previous stage, but in most cases a return to the flight planning stage is invoked for problem solving. It is here that a certain level of automation (e.g. Python scripting) is essential in order to quickly and accurately alter flight plan parameters in the field. Some examples of an iterative workflow, based on our experience, are provided here:

1. During the image quality check, image blur is detected (see Figure 7). The speed of the flight path is reduced (or camera shutter speed increased) and the mission is repeated after being reloaded to the UAV. 


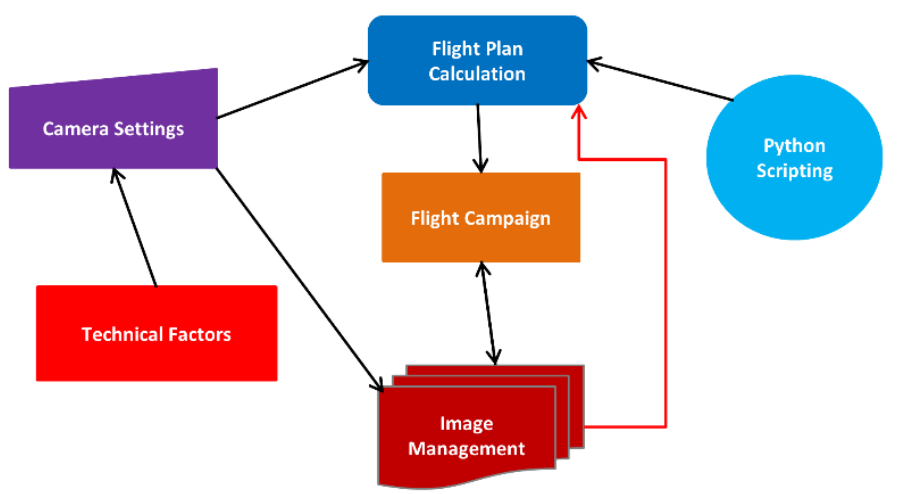

Figure 7: Flight speed vs. Shutter speed

2. During a mission, the angle of the gimbal is seen to be wrong (see Figure 8). The technical problem is solved (hardware or WP-event based), and the flight plan is simulated to test the gimbal's functionality. The flight mission is repeated.

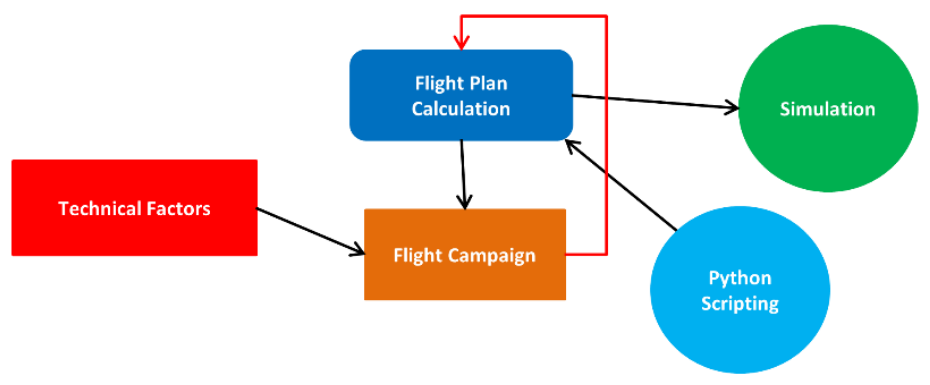

Figure 8: Faulty gimbal angle

3. A sudden change in wind speed or other atmospheric turbulence is detected and the UAV is having difficulty reaching waypoints (see Figures 5 and 9). The flight plan is altered, the waypoint radius is increased to $15 \mathrm{~m}$ (previously $10 \mathrm{~m}$ ), and the grid is changed from $\mathrm{N}$ to $\mathrm{S}$ (S to $\mathrm{N}$ ) lines to $\mathrm{E}$ to $\mathrm{W}$ (W to $\mathrm{E}$ ).

4. A variation could be that, alongside changing the flight direction, the waypoint events are removed, and the UAV flies the simplified grid lines, implementing the camera's timelapse function to trigger images in place of WP-event triggers. 


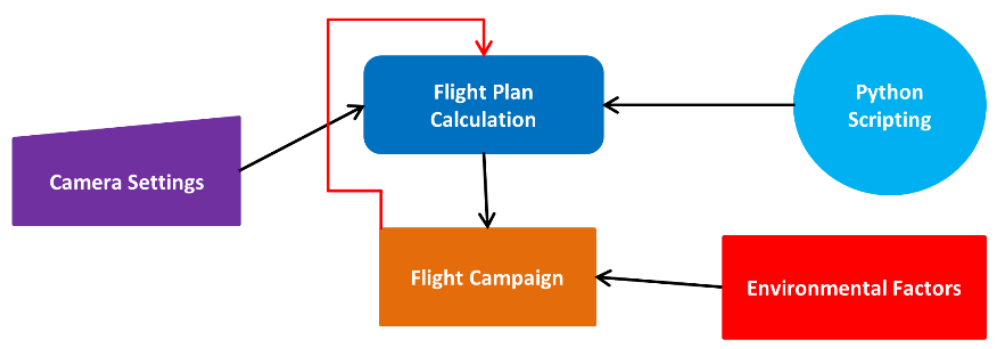

Figure 9: Strong winds

\section{Conclusion and Future Research}

The process of acquiring UAV-based imagery for the purpose of producing high-density point clouds can be optimized when an effective workflow is considered for flight campaigns. Degradation of image quality due to factors such as meteorological and physical influences can be handled through effective and iterative flight planning, and a certain level of flexibility through automation (scripting) in the field is essential. An iterative process to perform problem solving in the field was explored. It was shown that an optimized workflow is significantly dependent on the ability to efficiently alter flight plans during a flight campaign. A well-planned strategy to deal with unexpected occurrences during flight campaigns also increases the potential to save valuable time spent in the field and power capacity.

Developments are needed in flight-planning software, for example the capability to import editable text files containing flight grid parameters. Flight-planning software should also be capable of creating flight grids that can take into account oblique camera projections in order to ensure proper area coverage. Flying a grid designed for nadir images while using oblique camera angles can result in images outside the area of interest, and an inefficient flight mission, with increased pre-processing data management workloads. Furthermore, the possibility to design flight plans that can ensure proper coverage in mountainous areas is limited. Flight planning with a custom Digital Elevation Model derived from, for example, an existing LiDAR dataset could assist in establishing flying altitude variables. Additionally, flight plan grids calculated specifically for a LiDAR dataset could have a positive influence on the photogrammetric point cloud accuracy when applied to 3D-enhanced forest inventories.

Our research continues with the development of an open source tool to import flight planning grids and relevant waypoint event details into the KopterTool (MikroKopter) ground control station. The tool should also have the capability to convert Mikrokopterspecific wpl files into csv file format, in order to enable detailed flight planning for our Quadrocopter (Phantom 3) via the DJI ultimate flight app. Furthermore, we are exploring various UAV-based aerial image acquisition methods for uses in operational forestry and forestry research. We are particularly interested in assessing the accuracy of 3D forest-stand data, improving flight-planning strategies, as well as comparison studies of the empirical implementation of various remote sensing techniques for point cloud and orthomosaic analysis. 


\section{References}

Basgall, P. (2013). Lidar point cloud and stereo image point cloud fusion (Master's thesis, Naval Postgraduate School). Retrieved from http://www.dtic.mil/dtic/tr/fulltext/u2 /a589768.pdf

Campbell, J.B., \& Wynne, R.H. (2011). Introduction to remote sensing (5th ed.). New York, NY: The Guilford Press.

Colditz, C., Coconu, L., Deussen, O., \& Hege, C. (2005). Real-time rendering of complex photorealistic landscapes using hybrid level-of-detail approaches. Trends in Real-Time Landscape Visualization and Participation (pp. 97-106). Heidelberg, Germany: Wichmann Verlag, ISBN 387907-429-1.

DJI ultimate flight app. Retrieved from http://djiultimateflight.com/

Hirschmüller, H. (2011). Semi-global matching - motivation, developments and applications. 54th Photogrammetric Week, 173-184. Retrieved from http://www.ifp.unistuttgart.de/ publications/phowo11/180Hirschmueller.pdf

Hollaus, M. (2014). High resolution aerial images from UAV for forest applications state-of-the-art. The consortium of the project Interreg Alpine Space, Project number 2-3-2-FR. Retrieved from http://goo.gl/XXIbDY

Lisein J., Pierrot-deseilligny M., Bonnet S. \& Lejeune P. (2013). A photogrammetric workflow for the creation of a forest canopy height model from small unmanned aerial system imagery. Forests, 4 , 922-944. doi:10.3390/f4040922.

MikroKopter. Retrieved from http://www.mikrokopter.de/en/home

Miri. M., Bayer, S. \& Bucher, T. (2013). Evaluation of high resolution digital surface models for single tree extraction approaches in mixed forests, DGPF Tagungsband, 22. Retrieved from http://elib.dlr.de/82747/1/Miri-et-al_DGPF_Tagungsband_2013.pdf

Pix4D Support. Which factors affect the accuracy of the Automatic Aerial Triangulation (AAT)? Retrieved from https://support.pix4d.com/hc/en-us/articles/202559049-Which-factors-affectthe-accuracy-of-the-Automatic-Aerial-Triangulation-AAT-

Rosnell, T. \& Honkavaara E. (2012). Point cloud generation from aerial image data acquired by a quadrocopter type micro unmanned aerial vehicle and a digital still camera. Sensors, 12, 453-480. doi:10.3390/s120100453

Rosnell T., Honkavaara, E., \& Nurminen, K. (2012). On geometric processing of multi-temporal image data collected by light uav systems. International archives of the Photogrammetry, Remote Sensing and Spatial Information Sciences, Vol. XXXVIII-1/C22. Retrieved from http://www.geometh.ethz.ch/uav_g/proceedings/rosnell

Shahbazi M., Sohn, G.B., Théau, J., \& Ménard, P. (2015). UAV-based point cloud generation for open-pit mine modelling. The International Archives of the Photogrammetry, Remote Sensing and Spatial Information Sciences, Volume XL-1/W4.

Retrieved from http://www.int-arch-photogramm-remote-sens-spatial-inf-sci.net/XL-1W4/313/2015/isprsarchives-XL-1-W4-313-2015.pdf

St-onge B., Audet, F., \& Bégin, J. (2015). Characterizing the height structure and composition of a boreal forest using an individual tree crown approach applied to photogrammetric point clouds. Forests 2015, 6, 3899-3922.

White, J.C., Wulder, M.A., Vastaranta, M., Coops, N.C., Pitt, D., \& Woods, M. (2013). The utility of image-based point clouds for forest inventory: A comparison with airborne laser scanning. Forests 2013, 4, 518-536.

Wolf, P. R., Dewitt, B. A., \& Wilkinson, B.E. (2014). Elements of photogrammetry (4th ed.). New York, NY: McGraw-Hill, ISBN: 978-0-07-176111-6. 